\title{
ON LAPLACIAN SPECTRUM OF DENDRITE TREES
}

\author{
By Yuyang Xu and Jianfeng YaO \\ The University of Hong Kong
}

\begin{abstract}
For dendrite graphs from biological experiments on mouse's retinal ganglion cells, a paper by Nakatsukasa, Saito and Woei reveals a mysterious phase transition phenomenon in the spectra of the corresponding graph Laplacian matrices. While the bulk of the spectrum can be well understood by structures resembling starlike trees, mysteries about the spikes, that is, isolated eigenvalues outside the bulk spectrum, remain unexplained. In this paper, we bring new insights on these mysteries by considering a class of uniform trees. Exact relationships between the number of such spikes and the number of T-junctions are analyzed in function of the number of vertices separating the T-junctions. Using these theoretical results, predictions are proposed for the number of spikes observed in real-life dendrite graphs. Interestingly enough, these predictions match well the observed numbers of spikes, thus confirm the practical meaningness of our theoretical results.
\end{abstract}

1. Introduction. Dendrite graphs are an important data analysis tool for understanding biological functions of the retinal ganglion cells (RGCs) of a mouse [1]. Characteristic features of these graphs such as dendritic field area, dendrite lengths, or number of dendrites can be used to classify various RGCs into different groups which do have different biological functions. The details about data acquisition and their conversion to dendrite graphs, which are almost trees, can be found in [3]. Figure 1 illustrates two typical dendrite graphs of RGCs together with a plot of eigenvalues of their graph Laplacian. When Nakatsukasa et al. [2] analyzed the spectrum of the graph Laplacian of these dendrite graphs, they revealed a "mysterious" phase transition phenomenon in the spectrum. The eigenvalue distribution consists of a smooth bell-shaped curve ranging from 0 to 4 , the bulk, followed by a sudden jump at 4 and a few spikes, i.e., isolated eigenvalues greater than 4 . In order to understand this phenomenon, they considered the family of starlike trees, i.e., trees with exactly one vertex of degree greater than 2 , as a simplified model for these complex dendrite graphs. Actually, the bulk of the dendrite spectra is well approximated by such starlike trees. However, the mechanism that generates those spike eigenvalues remains unknown.

AMS 2000 subject classifications: Primary 15A18; Secondary 05C05

Keywords and phrases: Dendrite graphs, Eigenvalue distribution, Graph Laplacian, Retinal ganglion cells, Number of spikes, Spike eigenvalues 


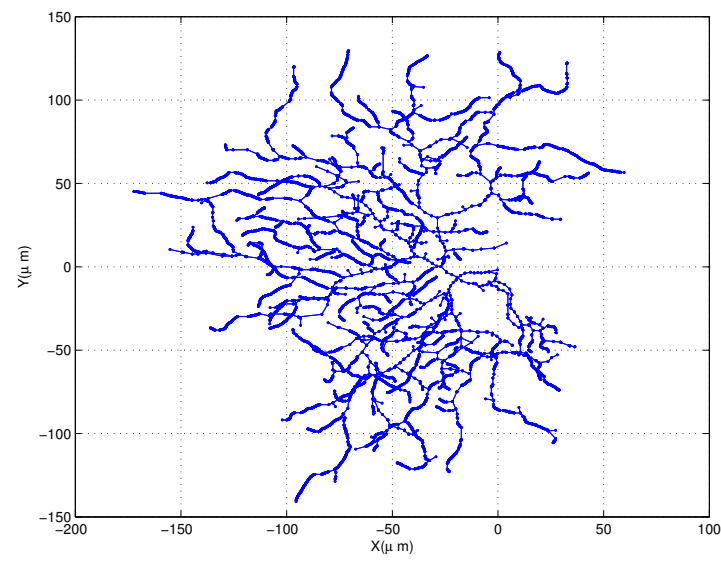

(a) RGC \#60

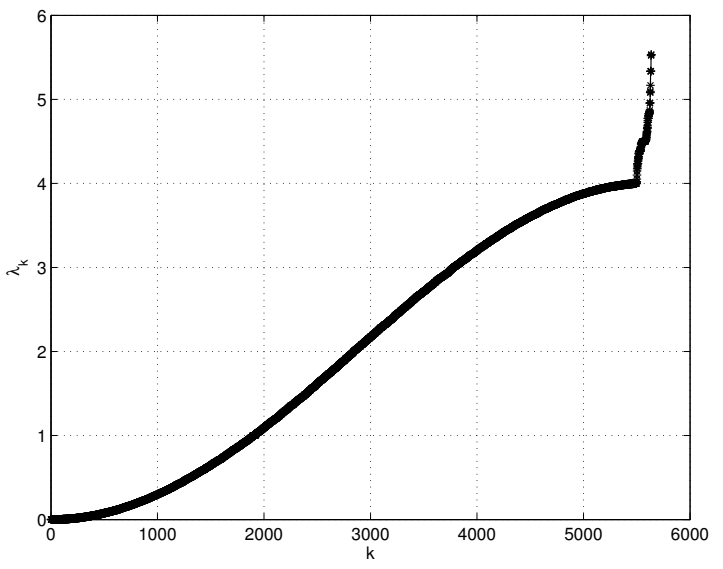

(c) Eigenvalues of (a)

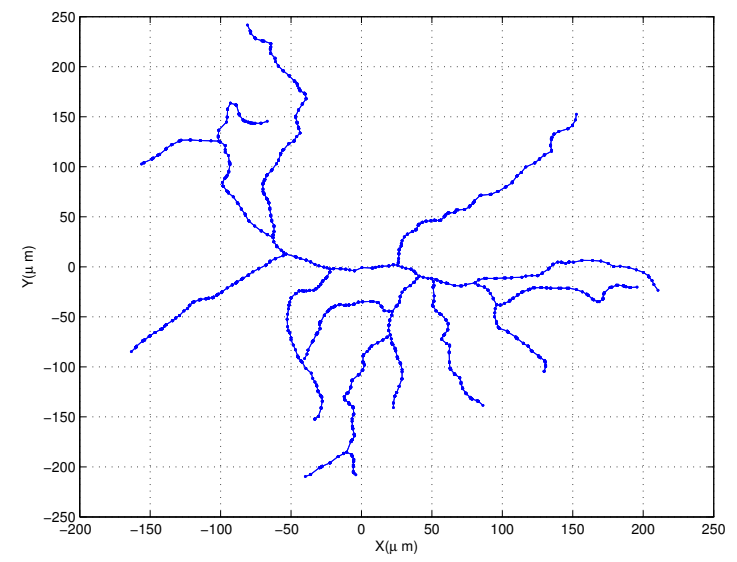

(b) RGC \#100

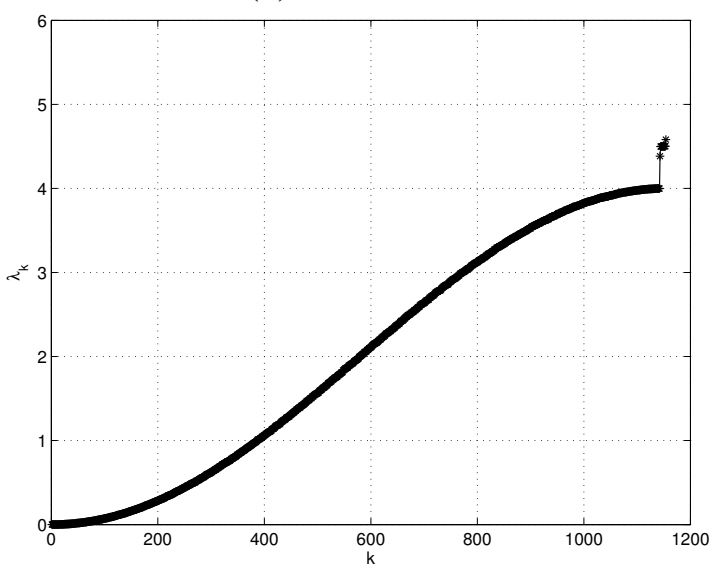

(d) Eigenvalues of (b)

FIG 1. (a) and (b): typical 2D projection of dendrites of retinal ganglion cells (RGCs) of a mouse; (c) and (d): eigenvalue distribution of corresponding graph Laplacians. Both graphs show a phase transitions occur at the value 4. Data and code used for producing the figure by courtesy of Prof. Naoki Saito (University of California, Davis). 


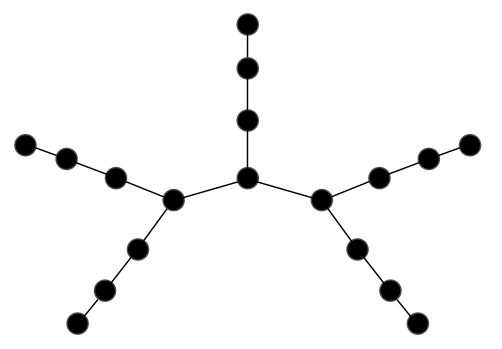

(a) $H_{0,3}$ with $n_{\mathrm{T}}=3$

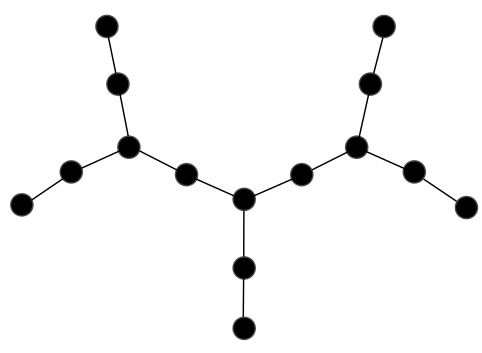

(b) $H_{1,2}$ with $n_{\mathrm{T}}=3$

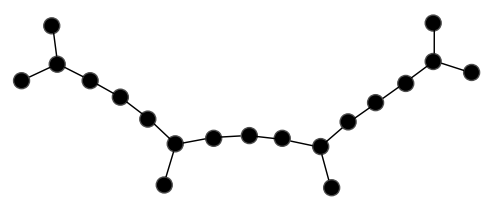

(c) $H_{3,1}$ with $n_{\mathrm{T}}=4$

FIG 2. Examples of uniform trees $H_{m, k}$ with trunk length $m$ and branch length $k$.

This paper shed new lights on the "mysteries around the graph Laplacian eigenvalue 4" found in [2]. We observe that dendrite trees consist of a number of T-junctions, whose degree are exactly 3 , a lot of path vertices, whose degree are exactly 2 , and pendant vertices, whose degree are 1. As a more involved model than starlike trees, we consider a class of uniform trees. Precisely, let $m \geqslant 0$ and $k \geqslant 1$ be nonnegative integers. A tree with maximum degree of 3 is a uniform tree of type $(m, k)$, denoted as $H_{m, k}$, if

1. the tree has a path lining a number of T-junctions (vertices of degree 3) where any two adjacent T-junctions are separated by a segment made with a given number of $m$ vertices, called trunk;

2. from each T-junction departs a branch which is a simple path with $k$ vertices terminating at a pendant vertex with degree 1 .

Some examples of uniform trees are given in Figure 2.

Our research starts from some numerical experiments on such uniform trees. To explain empirical findings from these numerical experiments, let us first introduce some notations. Consider an arbitrary graph $G=(V, E)$ with $n$ vertices in $V$ and edge set $E$. Let $\mathbf{L}(G)$ be its Laplacian matrix with eigenvalues $\lambda_{1}(G) \geqslant \cdots \geqslant \lambda_{j}(G) \geqslant \cdots \geqslant \lambda_{n}(G)=0$. Define

$$
n_{4^{+}}(G):=\#\left\{\lambda_{j}(G) \mid \lambda_{j}(G) \geqslant 4,1 \leqslant j \leqslant n\right\} \text {, i.e., the number of eigenvalues of } \mathbf{L}(G) \text { at }
$$
least equal to 4 ;

$n_{\mathrm{T}}(G):=\#\left\{v_{j} \in V \mid \operatorname{deg}\left(v_{j}\right)=3,1 \leqslant j \leqslant n\right\} \geqslant 2$, the number of T-junctions in $G$.

Our empirical findings of uniform trees $H_{m, k}$ are as follows:

(a) For any $H_{m, k}$ with trunk length $m \geqslant 2, n_{4^{+}}\left(H_{m, k}\right)=n_{\mathrm{T}}\left(H_{m, k}\right)$ for all $k \geqslant 1$;

(b) For $H_{m, k}$ with trunk length $m=1$ and any fixed T-junction number $n_{\mathrm{T}}(\cdot) \equiv n_{\mathrm{T}}\left(H_{m, k}\right)$, $n_{4^{+}}\left(H_{m, k}\right) \nearrow n_{\mathrm{T}}$ as $k \rightarrow \infty$;

(c) For $H_{m, k}$ with trunk length $m=0$ and any fixed T-junction number $n_{\mathrm{T}}(\cdot) \equiv n_{\mathrm{T}}\left(H_{m, k}\right)$, 
TABLE 1

Uniform trees with constant trunk length $m=1$ : numerical values of $n_{4^{+}}\left(H_{1, k}\right)$ for various branch lengths $k$ and T-junction numbers $n_{\mathrm{T}}$.

\begin{tabular}{|c|c|c|c|c|c|c|c|c|c|c|c|c|c|c|c|}
\hline$n_{4^{+}}\left(H_{1, k}\right)>n_{\mathrm{T}}$ & 2 & 3 & 4 & 5 & 6 & 7 & 8 & 9 & 10 & 15 & 20 & 25 & 30 & 40 & 50 \\
\hline 1 & 1 & 2 & 2 & 3 & 4 & 4 & 5 & 5 & 6 & 9 & 12 & 15 & 18 & 24 & 30 \\
\hline 2 & 2 & 2 & 3 & 4 & 5 & 5 & 6 & 7 & 7 & 11 & 14 & 18 & 21 & 28 & 36 \\
\hline 3 & & 3 & 3 & 4 & 5 & 6 & 6 & 7 & 8 & 12 & 15 & 19 & 23 & 31 & 38 \\
\hline 4 & & & 4 & 4 & 5 & 6 & 7 & 7 & 8 & 12 & 16 & 20 & 24 & 32 & 40 \\
\hline 5 & & & & 4 & 5 & 6 & 7 & 8 & 9 & 13 & 17 & 21 & 25 & 33 & 41 \\
\hline 6 & & & & 5 & 5 & 6 & 7 & 8 & 9 & 13 & 17 & 21 & 25 & 33 & 42 \\
\hline 7 & & & & & 5 & 6 & 7 & 8 & 9 & 13 & 17 & 21 & 25 & 34 & 42 \\
\hline 8 & & & & & 6 & 6 & 7 & 8 & 9 & 13 & 17 & 22 & 26 & 34 & 43 \\
\hline 9 & & & & & & 6 & 7 & 8 & 9 & 13 & 18 & 22 & 26 & 35 & 43 \\
\hline 10 & & & & & & 7 & 7 & 8 & 9 & 13 & 18 & 22 & 26 & 35 & 43 \\
\hline 15 & & & & & & & 8 & 8 & 9 & 14 & 18 & 23 & 27 & 36 & 45 \\
\hline 20 & & & & & & & & 9 & 9 & 14 & 18 & 23 & 27 & 37 & 46 \\
\hline 30 & & & & & & & & & 10 & 14 & 19 & 23 & 28 & 37 & 46 \\
\hline 50 & & & & & & & & & & 15 & 19 & 24 & 29 & 38 & 47 \\
\hline 100 & & & & & & & & & & & 20 & 24 & 29 & 39 & 48 \\
\hline 150 & & & & & & & & & & & & 25 & 29 & 39 & 49 \\
\hline 200 & & & & & & & & & & & & & 30 & 39 & 49 \\
\hline
\end{tabular}

TABLE 2

Uniform trees with constant trunk length $m=0$ : numerical values of $n_{4^{+}}\left(H_{0, k}\right)$ for various branch lengths $k$ and T-junction numbers $n_{\mathrm{T}}$.

\begin{tabular}{|c|c|c|c|c|c|c|c|c|c|c|c|c|}
\hline$\frac{n_{4^{+}}\left(H_{0, k}\right) \backslash n_{\mathrm{T}}}{k}$ & 2 & 3 & 4 & 5 & 6 & 7 & 8 & 9 & 10 & 15 & 30 & 50 \\
\hline 1 & 1 & 1 & 2 & 2 & 2 & 3 & 3 & 4 & 4 & 6 & 12 & 20 \\
\hline 2 & : & 2 & 2 & 2 & 3 & 3 & 4 & 4 & 5 & 7 & 13 & 22 \\
\hline 3 & $\vdots$ & $\vdots$ & $\vdots$ & 3 & 3 & 4 & 4 & 4 & 5 & 7 & 14 & 23 \\
\hline 4 & $\vdots$ & $\vdots$ & $\vdots$ & $\vdots$ & : & : & $\vdots$ & 5 & 5 & 7 & 14 & 24 \\
\hline 5 & $\vdots$ & : & $\vdots$ & $\vdots$ & : & : & $\vdots$ & $\vdots$ & $\vdots$ & 7 & 15 & 24 \\
\hline 6 & $\vdots$ & : & $\vdots$ & & $\vdots$ & & $\vdots$ & $\vdots$ & $\vdots$ & 8 & 15 & 24 \\
\hline 7 & $\vdots$ & $\vdots$ & $\vdots$ & : & : & : & $\vdots$ & $\vdots$ & $\vdots$ & $\vdots$ & $\vdots$ & 24 \\
\hline 8 & $\vdots$ & : & $\vdots$ & & & & $\vdots$ & $\vdots$ & $\vdots$ & $\vdots$ & & 25 \\
\hline 20 & $\vdots$ & $\vdots$ & $\vdots$ & : & : & $\vdots$ & $\vdots$ & $\vdots$ & $\vdots$ & $\vdots$ & $\vdots$ & $\vdots$ \\
\hline 100 & 1 & 2 & 2 & 3 & 3 & 4 & 4 & 5 & 5 & 8 & 15 & 25 \\
\hline
\end{tabular}

$$
n_{4^{+}}\left(H_{m, k}\right) \nearrow\left\lfloor\frac{n_{\mathrm{T}}+1}{2}\right\rfloor \text { as } k \rightarrow \infty .
$$

The convergences in (b) and (c) above can be verified with the numerical results given in Tables 1 and 2, respectively.

The main contribution of this paper is a rigorous proof for the three empirical findings (a)-(b)-(c) above.

These theoretical results on uniform trees provide a way to explain the spectra of the graph Laplacians of real-life dendrite trees as those shown in Figure 1. Indeed, these dendrite graphs can be viewed as a mixture of many uniform trees of different types, that is, with different trunk and branch lengths. Using the results (a)-(b)-(c), we provide in Section 4 an estimation method for the number of spikes in term of different T-junctions and corresponding trunk 
lengths observed in these dendrite graphs. Estimates resulting from this method are thus compared to the empirical values found in the dendrite graphs. Interestingly enough, the estimates match the empirical values very well.

The remaining of the paper is organized as follows. Section 2 recalls some known and useful results on Laplacian eigenvalues of trees and matrix perturbation. The main results of the paper are introduced in Section 3. As mentioned, we apply these theoretical results on uniform trees to estimate the number of spikes in dendrite graphs in Section 4.

2. Preliminary Results. A few known results on Laplacian eigenvalues of starlike trees and matrix perturbation will be useful. First recall that a starlike tree is defined as a tree with exactly one vertex of degree at least equal to 3 . So the smallest starlike tree is $K_{1,3}$, which is also known as a claw.

Lemma 2.1 (Y. Nakatsukasa et al. [2]). A starlike tree has exactly one Laplacian eigenvalue greater than or equal to 4 . The equality holds if and only if the starlike tree is $K_{1,3}$.

There is a well-known upper bound for the number of Laplacian spikes for trees where vertices have degree 3 at most.

Lemma 2.2 (Y. Nakatsukasa et al. [2]). For any finite tree $G$ with maximum degree of 3, we have

$$
n_{4^{+}}(G) \leqslant n_{\mathrm{T}}(G)
$$

Next is a very useful interlacing lemma for perturbation on Laplacian eigenvalues.

Lemma 2.3 (R. Grone et al. [4]). Let $G$ be a tree on $n$ vertices. Suppose $\widetilde{G}$ is a subtree of $G$ obtained by removing exactly one edge, then the $n-1$ largest eigenvalues of $\mathbf{L}(\widetilde{G})$ interlaces the eigenvalues of $\mathbf{L}(G)$. That is,

$$
\lambda_{1}(G) \geqslant \lambda_{1}(\widetilde{G}) \geqslant \lambda_{2}(G) \geqslant \cdots \geqslant \lambda_{n-1}(G) \geqslant \lambda_{n-1}(\widetilde{G})=\lambda_{n}(G)=0
$$

An important consequence of this interlacing lemma on trees is as follows. Consider a tree $G$ and a pendant vertex $s$ of $G$, that is, the degree of $s$ is 1 . Let $\widetilde{G}$ be the sub-tree obtained from $G$ by removing $s$ and the edge terminating at $s$. Then $n_{4^{+}}(\widetilde{G}) \leqslant n_{4^{+}}(G)$. Consequently $n_{4^{+}}\left(H_{m, k}\right)$ is increasing in the branch length $k$. Taking into account the upper bound $n_{\mathrm{T}}(G)$ in Lemma 2.2, if we let both the number of T-junctions $n_{\mathrm{T}}(\cdot)$ and the trunk length $m$ be fixed and the branch length $k$ tending to infinity, it holds that

$$
\lim _{k \rightarrow \infty} n_{4^{+}}\left(H_{m, k}\right)=\ell=\ell\left(m, n_{\mathrm{T}}(\cdot)\right) \leqslant n_{\mathrm{T}}(\cdot),
$$


for a limit $\ell$ depending on $m$ and $n_{\mathrm{T}}(\cdot)$.

The following result on tridiagonal matrices will be also useful.

Lemma 2.4 (W. C. Yueh et al. [5]). Consider a tridiagonal matrix

$$
\mathbf{A}_{n}=\left(\begin{array}{rrrrrr}
\gamma+b & c & & & \\
& a & b & \ddots & & \\
& \ddots & \ddots & \ddots & \\
& & \ddots & \ddots & \ddots & \\
& & & \ddots & b & c \\
& & & & a & b+\delta
\end{array}\right)_{n \times n}
$$

where $a, b, c, \gamma, \delta \in \mathbb{C}, a c \neq 0$ and $n \geqslant 2$. The eigenvalues (unordered) of $\mathbf{A}_{n}$ are given by

$$
\lambda_{j}=b+2 \sqrt{a} \sqrt{c} \cos \theta_{j}, \quad j=1,2, \ldots, n,
$$

where $\theta_{j} \in \mathbb{C}$ satisfy

$$
\begin{cases}a c \sin (n+1) \theta_{j}-\sqrt{a} \sqrt{c}(\gamma+\delta) \sin n \theta_{j}+\gamma \delta \sin (n-1) \theta_{j}=0 ; & \text { if } \sin \theta_{j} \neq 0 \\ a c \cdot(n+1)-\cos \theta_{j} \sqrt{a} \sqrt{c}(\gamma+\delta) \cdot n+\gamma \delta \cdot(n-1)=0 ; & \text { if } \sin \theta_{j}=0\end{cases}
$$

In particular, when $a, b, c, \gamma, \delta$ are all real and $a=c, \mathbf{A}_{n}$ is real symmetric thus with real eigenvalues. By Equation (2.2), we have all $\theta_{j} \in \mathbb{R}$.

Also, note that for a path graph $P_{n}$ with $n$ vertices, i.e., a tree with maximum degree of 2 , the Laplacian matrix is

$$
\mathbf{L}\left(P_{n}\right)=\left(\begin{array}{ccccccc}
1 & & -1 & & & & \\
-1 & & 2 & \ddots & & & \\
& \ddots & \ddots & \ddots & & \\
& & \ddots & \ddots & \ddots & \ddots & \\
& & & \ddots & \ddots & \ddots & \\
& & & \ddots & 2 & -1 \\
& & & & -1 & 1
\end{array}\right)_{n \times n},
$$

which is a special case of $\mathbf{A}_{n}$ in Lemma 2.4. Applying the lemma we recover the well-known Laplacian eigenvalues of $P_{n}$ given by

$$
\lambda_{j}\left(P_{n}\right)=4 \sin ^{2}\left(\frac{n-j}{2 n} \pi\right), \quad j=1,2, \ldots, n .
$$

They are all less than 4.

Finally, we recall the classical Schur complement formula.

Lemma 2.5 (Schur Complement Formula). For an $n \times n$ real matrix $\mathbf{A}$, define $\mathbf{A}_{j}, j=$ $1,2, \ldots, n$, to be the $j$-th major submatrix of order $n-1$, i.e., the submatrix resulting from $\mathbf{A}$ by deleting its $j$-th row and $j$-th column. Suppose both $\mathbf{A}$ and $\mathbf{A}_{j}$ are nonsingular, then

$$
a^{j j}=\frac{1}{a_{j j}-\boldsymbol{\alpha}_{j}^{\top} \mathbf{A}_{j}^{-1} \boldsymbol{\beta}_{j}},
$$




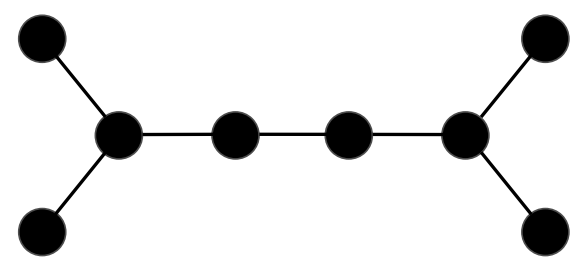

(a) $H_{2+}$

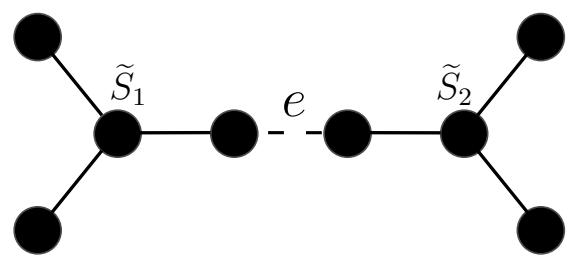

(b) $\widetilde{H}$

FIG 3. Illustration of base case in the proof of Theorem 3.1. (a) $H_{2}+$ with $n_{\mathrm{T}}=2$; (b) $\widetilde{H}$ and its two connected components $\widetilde{S}_{1}, \widetilde{S}_{2}$, obtained from $H_{2}$ by the removal of edge $e$.

where $a^{j j}$ and $a_{j j}$ are the $j$-th diagonal entry of $\mathbf{A}^{-1}$ and $\mathbf{A}$, respectively; $\boldsymbol{\alpha}_{j}^{\top}$ is the vector obtained from the $j$-th row of $\mathbf{A}$ by deleting its $j$-th entry, and $\boldsymbol{\beta}_{j}$ is the vector obtained from the $j$-th column of $\mathbf{A}$ by deleting its $j$-th entry.

3. Main Results. Throughout this section, we consider a uniform tree with all its Tjunctions aligned on a single line. Whenever no ambiguity is possible about the branch length $k$, we simply use $H_{m}$ to denote $H_{m, k}$ for $m=0,1$ and $H_{2^{+}}$for $m \geqslant 2$.

\subsection{Uniform trees with trunk length $m \geqslant 2$.}

TheOREM 3.1. For uniform trees with trunk length $m \geqslant 2$, we have

$$
n_{4^{+}}\left(H_{m, k}\right)=n_{\mathrm{T}}\left(H_{m, k}\right), \quad \forall k \geqslant 1 .
$$

Proof. The result is proved by induction on $n_{\mathrm{T}}$.

$\diamond$ Base Case When $n_{\mathrm{T}}=2$, let $\widetilde{H}=(V, E \backslash\{e\})$, where $e$ is an edge on the trunk in $H_{2^{+}}$that is not incident to any $\mathrm{T}$-junction. The existence of such $e$ is guaranteed by the assumption that $m \geqslant 2$, thus at least three edges lie between two consecutive T-junctions. Then $\widetilde{H}$ consists of

two connected components, say $\widetilde{S}_{1}$ and $\widetilde{S}_{2}$, which are both starlike trees. Figure 3 shows an example of $H_{2+}$ and $\widetilde{H}$ with corresponding $\widetilde{S}_{1}, \widetilde{S}_{2}$ and an edge $e$ removed from $H_{2^{+}}$.

By Lemma 2.1, we have

$$
\lambda_{1}\left(\widetilde{S}_{1}\right) \geqslant 4>\lambda_{2}\left(\widetilde{S}_{1}\right), \quad \lambda_{1}\left(\widetilde{S}_{2}\right) \geqslant 4>\lambda_{2}\left(\widetilde{S}_{2}\right) .
$$

Without loss of generality, assume $\lambda_{1}\left(\widetilde{S}_{1}\right) \geqslant \lambda_{1}\left(\widetilde{S}_{2}\right)$. Then we will have

$$
\lambda_{1}(\widetilde{H})=\lambda_{1}\left(\widetilde{S}_{1}\right) \geqslant 4, \quad \lambda_{2}(\widetilde{H})=\lambda_{1}\left(\widetilde{S}_{2}\right) \geqslant 4
$$

Note that by Lemma 2.3, we have

$$
\lambda_{1}\left(H_{2^{+}}\right) \geqslant \lambda_{1}(\widetilde{H}) \geqslant \lambda_{2}\left(H_{2^{+}}\right) \geqslant \lambda_{2}(\widetilde{H}) .
$$


Using the result from (3.1), we get

$$
\lambda_{1}\left(H_{2^{+}}\right) \geqslant \lambda_{1}\left(\widetilde{S}_{1}\right) \geqslant \lambda_{2}\left(H_{2^{+}}\right) \geqslant \lambda_{1}\left(\widetilde{S}_{2}\right) \geqslant 4
$$

Hence, $n_{4^{+}} \geqslant 2$. However, by Lemma 2.2 , we have $n_{4^{+}} \leqslant n_{\mathrm{T}}=2$. Therefore, $n_{4^{+}}=n_{\mathrm{T}}$ in this case.

$\diamond$ Induction step $\quad$ Suppose $n_{4^{+}}=n_{\mathrm{T}}$ holds for all $n_{\mathrm{T}} \leqslant i$, for some $i \geqslant 2$.

When $n_{\mathrm{T}}=i+1$, similarly, let $\widetilde{H}=(V, E \backslash\{e\})$, where $e$ is an edge on a trunk in $H_{2^{+}}$and is not incident to any T-junction. Let $\widetilde{H}_{1}$ and $\widetilde{H}_{2}$ be the two connected components of $\widetilde{H}$, with $t_{1}$ and $t_{2}$ T-junctions, respectively. By inductive assumption we have

$$
\begin{aligned}
& \lambda_{1}\left(\widetilde{H}_{1}\right) \geqslant \cdots \geqslant \lambda_{t_{1}}\left(\widetilde{H}_{1}\right) \geqslant 4>\lambda_{t_{1}+1}\left(\widetilde{H}_{1}\right), \\
& \lambda_{1}\left(\widetilde{H}_{2}\right) \geqslant \cdots \geqslant \lambda_{t_{2}}\left(\widetilde{H}_{2}\right) \geqslant 4>\lambda_{t_{2}+1}\left(\widetilde{H}_{2}\right) .
\end{aligned}
$$

Note that $t_{1}+t_{2}=n_{\mathrm{T}}=i+1$. By Lemma 2.3, we have

$$
\lambda_{1}\left(H_{2^{+}}\right) \geqslant \lambda_{1}(\widetilde{H}) \geqslant \cdots \geqslant \lambda_{i+1}\left(H_{2^{+}}\right) \geqslant \lambda_{i+1}(\widetilde{H}) \geqslant 4 .
$$

Hence, $n_{4^{+}} \geqslant i+1$. However, by Lemma 2.2 we have $n_{4^{+}} \leqslant n_{\mathrm{T}}=i+1$. Hence $n_{4^{+}}=n_{\mathrm{T}}$.

3.2. Uniform trees with trunk length $m=1$. Unlike the previous situation with trunk length $m \geqslant 2$, numerical results in Table 1 shows that $n_{4^{+}}\left(H_{1, k}\right) \neq n_{\mathrm{T}}\left(H_{1, k}\right)$ may happen when $m=1$. The same table also shows that $n_{4^{+}}$approaches $n_{\mathrm{T}}$ as the branch length increases.

THEOREM 3.2. Consider uniform trees with trunk length $m=1$. When all the branches of $H_{1}$ are sufficiently long, i.e., when $k$ is large enough, we have

$$
n_{4^{+}}\left(H_{1, k}\right)=n_{\mathrm{T}}\left(H_{1, k}\right)
$$

Proof. For simplicity, we will use $t$ instead of $n_{\mathrm{T}}$, and denote the Laplacian matrix of a uniform tree $H_{1}$ by $\mathbf{L}_{1}$. By considering first the main path connecting all the T-junctions and then the branch(es) on each T-junction, the Laplacian matrix $\mathbf{L}_{1}$ has the form (see Figure 4)

$$
\mathbf{L}_{1}=\left(\begin{array}{ccccc}
\mathbf{A} & \mathbf{E}_{1}^{\top} & \mathbf{E}_{2}^{\top} & \cdots & \mathbf{E}_{t+2}^{\top} \\
\mathbf{E}_{1} & \mathbf{B} & & & \\
\mathbf{E}_{2} & & \mathbf{B} & & \\
\vdots & & & \ddots & \\
\vdots & & & \ddots & \\
\mathbf{E}_{t+2} & & & & \mathbf{B}
\end{array}\right),
$$




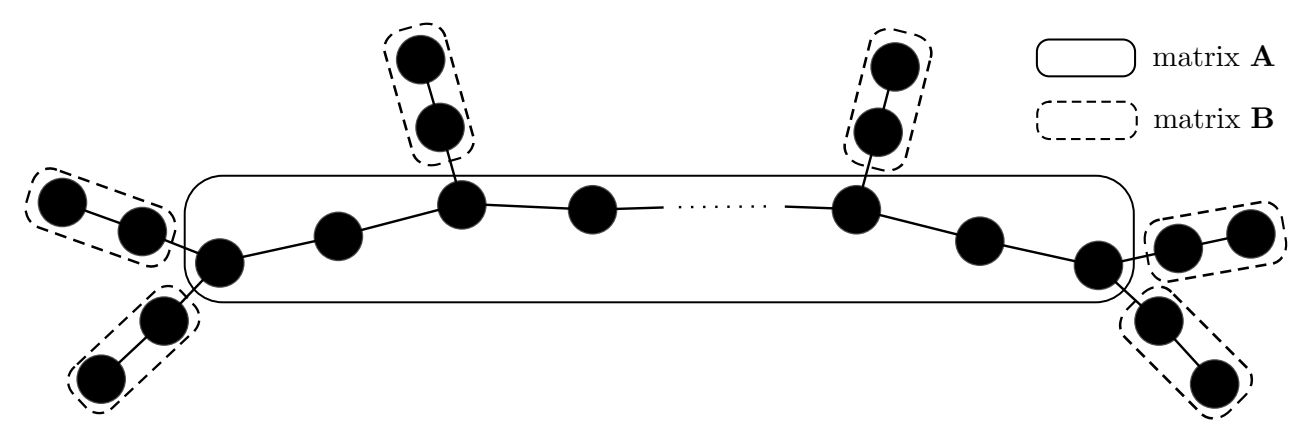

FIG 4. Illustration of uniform tree $H_{1, k}$ and its counterpart in $\mathbf{L}_{1}$, with $n_{\mathrm{T}}=4$.

where

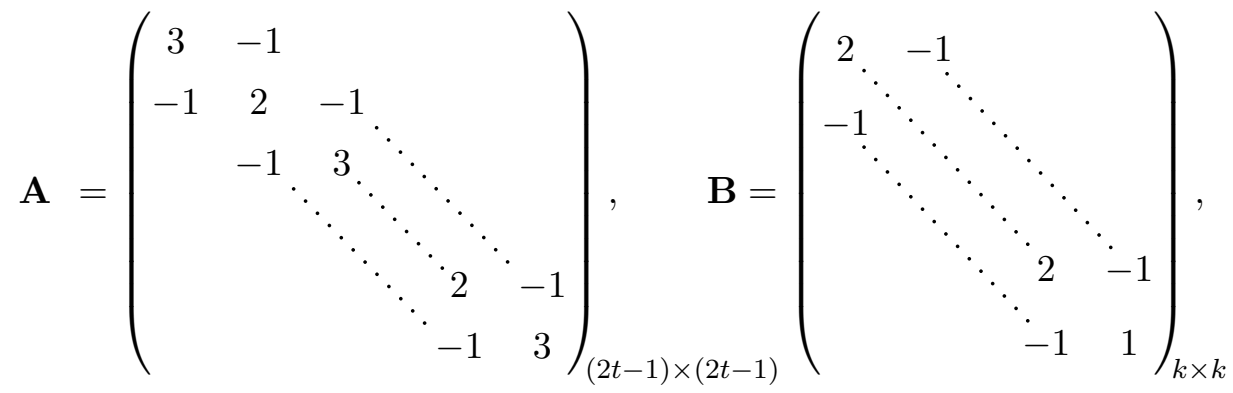

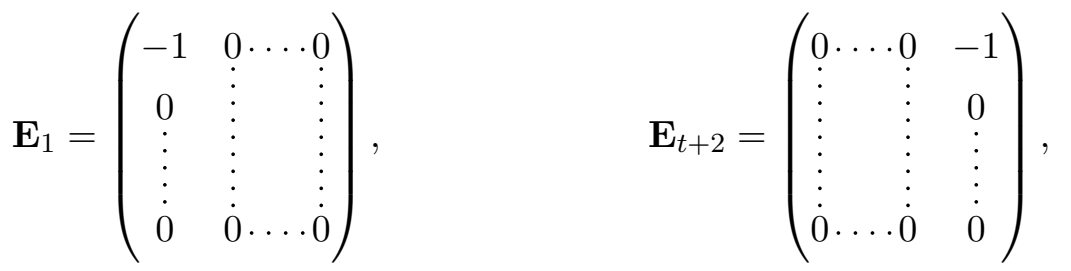

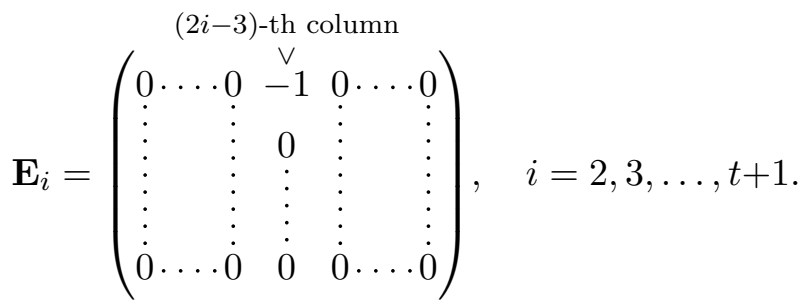

We can also write $\mathbf{L}_{1}$ in a more compact form, namely

$$
\mathbf{L}_{1}=\left(\begin{array}{cc}
\mathbf{A} & \mathbf{E}^{\top} \\
\mathbf{E} & \underset{\sim}{\mathbf{B}}
\end{array}\right),
$$

where

$$
\underset{\sim}{\mathbf{B}}=\mathbf{B} \otimes \mathbf{I}_{t+2}=\left(\begin{array}{cccc}
\mathbf{B} & & & \\
& \ddots & \\
& & \ddots & \\
& & & \\
& & & \mathbf{B}
\end{array}\right), \quad \mathbf{E}=\left(\begin{array}{c}
\mathbf{E}_{1} \\
\vdots \\
\vdots \\
\mathbf{E}_{t+2}
\end{array}\right)
$$

- Step 1: block decomposition of the determinant. Using Schur's determinant identity, any 
eigenvalue $\lambda$ of the Laplacian $\mathbf{L}_{1}$ (this is also $\lambda\left(H_{1}\right)$ ) solves the equation

$$
\begin{aligned}
0=\left|\lambda \mathbf{I}-\mathbf{L}_{1}\right| & =\left|\begin{array}{cc}
\lambda \mathbf{I}-\mathbf{A} & -\mathbf{E}^{\top} \\
-\mathbf{E} & \lambda \mathbf{I}-\underset{\sim}{\mathbf{B}}
\end{array}\right| \\
& =|\lambda \mathbf{I}-\underset{\sim}{\mathbf{B}}| \cdot\left|\lambda \mathbf{I}-\mathbf{A}-\mathbf{E}^{\top}(\lambda \mathbf{I}-\mathbf{B})^{-1} \mathbf{E}\right| \\
& =|\lambda \mathbf{I}-\mathbf{B}|^{t+1} \cdot\left|\lambda \mathbf{I}-\mathbf{A}-\sum_{i=1}^{t+2} \mathbf{E}_{i}^{\top}(\lambda \mathbf{I}-\mathbf{B})^{-1} \mathbf{E}_{i}\right| \\
& \equiv|\lambda \mathbf{I}-\mathbf{B}|^{t+1} \cdot|\lambda \mathbf{I}-\mathbf{A}-\mathbf{C}| .
\end{aligned}
$$

Here the factorization is valid for any $\lambda$ such that $\lambda \mathbf{I}-\underset{\sim}{\mathbf{B}} \neq 0$. Because by Lemma 2.4, we can obtain the eigenvalues of $\mathbf{B}$, i.e.,

$$
\lambda_{j}=2+2 \cos \frac{2 j \pi}{2 k+1}, \quad j=1,2, \ldots, k,
$$

which are all less than 4 . Therefore, to find those spike eigenvalues $\lambda\left(H_{1}\right) \geqslant 4$, it suffices to consider roots of equation $|\lambda \mathbf{I}-\mathbf{A}-\mathbf{C}|=0$.

To further expand $\mathbf{C}$, let $\varepsilon_{l}$ be the $l$-th canonical vector of $\mathbb{R}^{2 t-1}$, then we have the decomposition

$$
\mathbf{E}_{i}=-\varepsilon_{\sigma_{i}} \boldsymbol{\mu}_{1}^{\top}
$$

where

$$
\boldsymbol{\mu}_{1}=(1,0, \ldots, 0)^{\top} \in \mathbb{R}^{k}
$$

and

$$
\sigma_{i}= \begin{cases}1, & i=1 \\ 2 i-3, & 2 \leqslant i \leqslant t+1 \\ 2 t-1, & i=t+2\end{cases}
$$

is the column position of -1 in $\mathbf{E}_{i}$. Therefore,

$$
\begin{aligned}
& \mathbf{C}=\sum_{i=1}^{t+2} \varepsilon_{\sigma_{i}}\left(\boldsymbol{\mu}_{1}^{\top}(\lambda \mathbf{I}-\mathbf{B})^{-1} \boldsymbol{\mu}_{1}\right) \boldsymbol{\varepsilon}_{\sigma_{i}}^{\top} \\
& =\left(\boldsymbol{\mu}_{1}^{\top}(\lambda \mathbf{I}-\mathbf{B})^{-1} \boldsymbol{\mu}_{1}\right) \cdot \sum_{i=1}^{t+2} \varepsilon_{\sigma_{i}} \varepsilon_{\sigma_{i}}^{\top} \\
& =\alpha_{k}(\lambda) \cdot\left(\begin{array}{cccccc}
2 & & & & \\
& 0 & & & \\
& & 1 & & \\
& & & \ddots & & \\
& & & & 0 & \\
& & & & 2
\end{array}\right)_{(2 t-1) \times(2 t-1)}
\end{aligned}
$$


where $\alpha_{k}(\lambda)=\boldsymbol{\mu}_{1}^{\top}(\lambda \mathbf{I}-\mathbf{B})^{-1} \boldsymbol{\mu}_{1}$ is the top-left element of $(\lambda \mathbf{I}-\mathbf{B})^{-1}$.

To see what $\alpha_{k}(\lambda)$ really is, let us consider the sequence $\left(\alpha_{k}\right)_{k \geqslant 1} \equiv\left(\alpha_{k}(\lambda)\right)_{k \geqslant 1}$. By Lemma 2.5 we have the following recursion:

$$
\left\{\begin{array}{l}
\alpha_{k}=\frac{1}{\lambda-2-\alpha_{k-1}}, \quad k \geqslant 1 \\
\alpha_{0}=-1
\end{array} .\right.
$$

Let

$$
x=\frac{1}{2}(\lambda-2-\sqrt{\lambda(\lambda-4)}), \quad y=\frac{1}{2}(\lambda-2+\sqrt{\lambda(\lambda-4)}) .
$$

Then the solution to the recursion can be written in the following form,

$$
\alpha_{k}= \begin{cases}\frac{x^{k} y(x+1)-x y^{k}(y+1)}{x^{k}(x+1)-y^{k}(y+1)} \in\left(0, \frac{2 k-1}{2 k+1}\right), & \lambda>4 \\ \frac{2 k-1}{2 k+1}<1, & \lambda=4 .\end{cases}
$$

- Step 2: let $k \rightarrow \infty$ to find a full factorization of the limiting determinant.

With the preparation above, we can now let $k \rightarrow \infty$, i.e., we investigate the uniform tree $H_{1, k}$ when branch length tends to infinity. This limiting tree is denoted by $H_{1, \infty}$. Our remaining task is to show

$$
\#\left\{\lambda_{j} \mid \lambda_{j}\left(H_{1, \infty}\right)>4,1 \leqslant j \leqslant n\right\}=t .
$$

Suppose for a moment that this claim is true. By continuity of $H_{1, k} \mapsto \lambda_{j}\left(H_{1, k}\right)$, the above equation implies existence of a $k_{0}$ such that for all $k^{\prime} \geqslant k_{0}$, we have

$$
\begin{aligned}
t & =\#\left\{\lambda_{j} \mid \lambda_{j}\left(H_{1, \infty}\right)>4,1 \leqslant j \leqslant n\right\} \\
& =\#\left\{\lambda_{j} \mid \lim _{k \rightarrow \infty} \lambda_{j}\left(H_{1, k}\right)>4,1 \leqslant j \leqslant n\right\} \\
& =\#\left\{\lambda_{j} \mid \lambda_{j}\left(H_{1, k^{\prime}}\right)>4,1 \leqslant j \leqslant n\right\} \\
& \leqslant n_{4^{+}}\left(H_{1, k^{\prime}}\right) .
\end{aligned}
$$

By Lemma 2.2, $n_{4^{+}}\left(H_{1, k^{\prime}}\right) \leqslant t$. Hence $n_{4^{+}}\left(H_{1, k^{\prime}}\right)=t$ and our theorem is proved.

It remains to establish the claim (3.2). Note that when $k \rightarrow \infty$,

$$
\alpha_{k} \rightarrow \alpha:=\frac{1}{2}(\lambda-2-\sqrt{\lambda(\lambda-4)}) \in(0,1], \quad \lambda \geqslant 4 .
$$


So we have

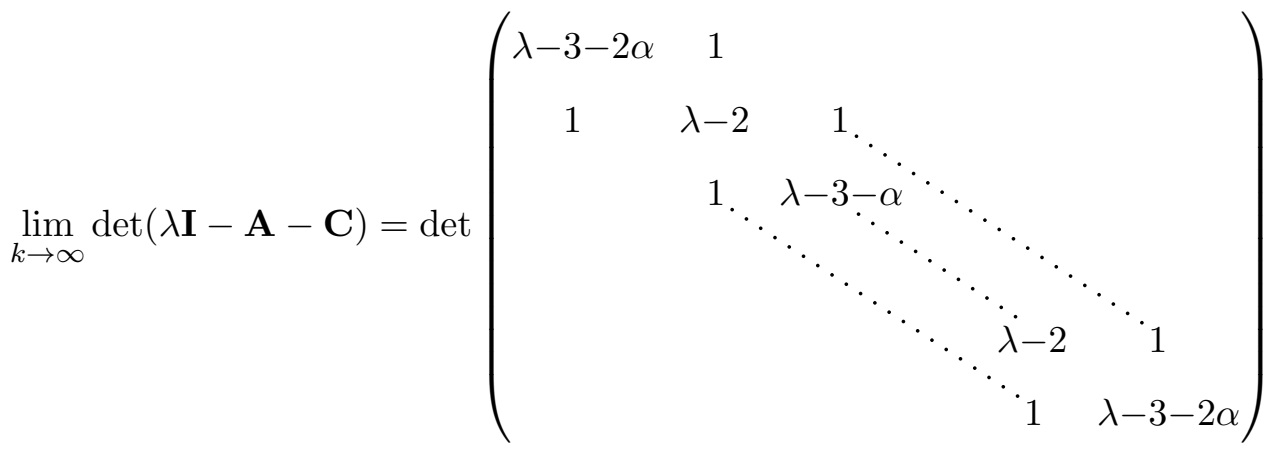

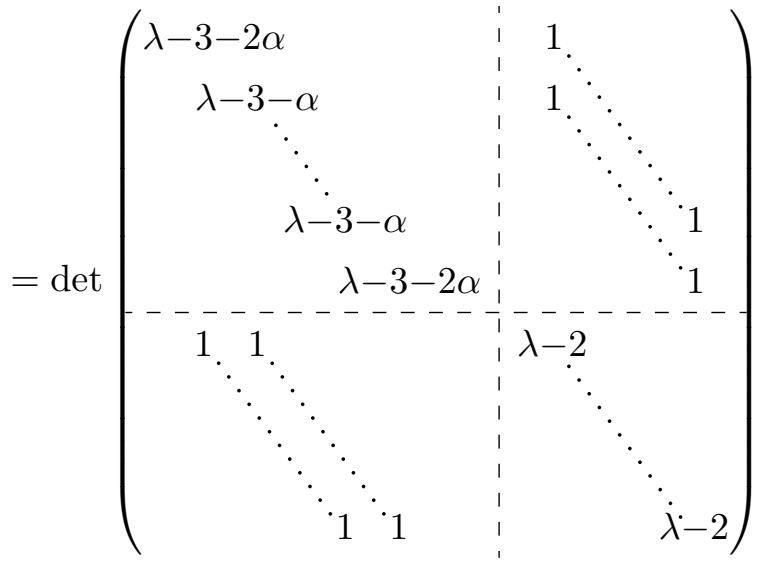

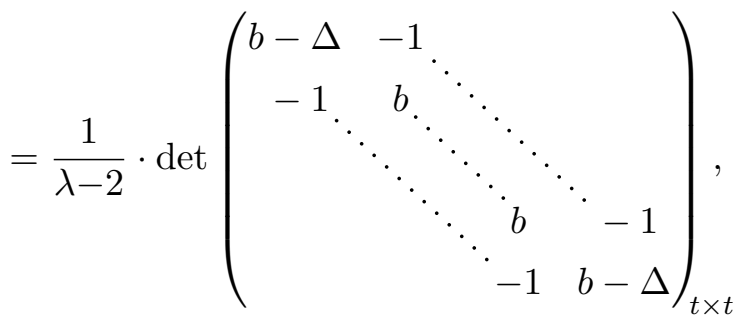

where

$$
b=(\lambda-2)(\lambda-3-\alpha)-2, \quad \Delta=\alpha(\lambda-2)-1,
$$

and Equation (3.3) is obtained by Schur's determinant identity. Note that both $b$ and $\Delta$ depend on $\lambda$.

Denote the matrix in Equation (3.3) by $\mathbf{T}$. To find the roots of equation $\operatorname{det} \mathbf{T}=0$, let

$$
\mathrm{g}(\lambda, \theta):=\sin (t+1) \theta-2 \Delta \sin t \theta+\Delta^{2} \sin (t-1) \theta
$$

and $\mu_{l}(\lambda), l=1,2, \ldots, t$ be eigenvalues of matrix $\mathbf{T}$, viewing $\lambda$ as a parameter. When $\lambda \geqslant 4$, $\mathbf{T}$ is real symmetric, by Lemma 2.4 , we have

$$
\operatorname{det} \mathbf{T}=\prod_{l=1}^{t} \mu_{l}(\lambda)=\prod_{l=1}^{t}\left(b-2 \cos \theta_{l}\right),
$$


where $\theta_{l} \equiv \theta_{l}(\lambda) \in \mathbb{R}$ is given by

$$
\begin{cases}\mathrm{g}\left(\lambda, \theta_{l}\right)=0, & \text { if } \sin \theta_{l} \neq 0 \text { and } \lambda>4 \\ \cos \theta_{l}=1, & \text { if } \sin \theta_{l}=0 \text { and } \lambda=4\end{cases}
$$

Without loss of generality, we can restrict $\theta_{l} \in(0, \pi)$ as we are considering $\lambda>4$.

With Equation (3.5), we factorized $\operatorname{det} \mathbf{T}$, which is essentially a polynomial of $\lambda$ of order $2 t$. Thus the number of our desired $\lambda$ can be obtained by counting roots from lower order polynomials. Formally speaking, we solve for

$$
\mu_{l}(\lambda)=b(\lambda)-2 \cos \theta_{l}=0,
$$

with restriction

$$
\mathrm{g}\left(\lambda, \theta_{l}\right)=0, \quad \theta_{l} \in(0, \pi)
$$

and count all the roots greater than 4 .

- Step 3: find $t$ number of $\lambda\left(H_{1, \infty}\right)$ 's that are greater than 4 .

Differentiating $\mu_{l}(\lambda)$ gives

$$
\frac{\mathrm{d} \mu_{l}}{\mathrm{~d} \lambda}=\lambda-3+\sqrt{\lambda(\lambda-4)}+\frac{2}{\sqrt{\lambda(\lambda-4)}}+2 \sin \theta_{l} \frac{\mathrm{d} \theta_{l}}{\mathrm{~d} \lambda} .
$$

Note that when $\lambda>4$, the left hand side of the above equation are all positive except for the last term. Also note that

$$
\begin{aligned}
\mu_{l}(4) & =-2-2 \cos \theta_{l}(4)<0, \\
\left.\mu_{l}(2 \sqrt{2}+2)\right) & =2-2 \cos \theta_{l}(2 \sqrt{2}+2)>0 .
\end{aligned}
$$

In the following we will consider function $\theta_{l}(\lambda) \in(0, \pi)$ determined by $\mathrm{g}\left(\lambda, \theta_{l}(\lambda)\right)=0$, and show that $\theta_{l}^{\prime}(\lambda)>0$, thus exactly $t$ number of $\lambda_{j}\left(H_{1, \infty}\right)$ 's that are strictly greater than 4 can be found. Observe that

$$
\begin{aligned}
\mathrm{g}(\lambda, \theta) & =\Im\left(\mathrm{e}^{\mathrm{i}(t+1) \theta}-2 \Delta \mathrm{e}^{\mathrm{i} t \theta}+\Delta^{2} \mathrm{e}^{\mathrm{i}(t-1) \theta}\right) \\
& =\Im\left(\mathrm{e}^{\mathrm{i} t \theta}\left(\mathrm{e}^{\mathrm{i} \theta}-2 \Delta+\Delta^{2} \mathrm{e}^{-\mathrm{i} \theta}\right)\right) .
\end{aligned}
$$

Let

$$
\rho \mathrm{e}^{\mathrm{i} \omega} \equiv\left(\mathrm{e}^{\mathrm{i} \theta}-2 \Delta+\Delta^{2} \mathrm{e}^{-\mathrm{i} \theta}\right)
$$

where $\rho$ is a positive number (obviously it cannot be 0 since $\Delta \in \mathbb{R}$ when $\lambda>4$ ) and $\omega \in[0,2 \pi$ ) is some function of $\Delta$ and $\theta$. Then

$$
\mathrm{g}(\lambda, \theta)=\Im\left(\mathrm{e}^{\mathrm{i} t \theta} \cdot \rho \mathrm{e}^{\mathrm{i} \omega}\right)=\rho \sin (t \theta+\omega) .
$$


So $g\left(\lambda, \theta_{l}\right)=0$ implies $t \theta_{l}+\omega=\mathbb{Z} \pi$. Differentiating this equation with respect to $\lambda$, we obtain i.e.,

$$
t \frac{\mathrm{d} \theta_{l}}{\mathrm{~d} \lambda}+\frac{\partial \omega}{\partial \theta_{l}} \frac{\mathrm{d} \theta_{l}}{\mathrm{~d} \lambda}+\frac{\partial \omega}{\partial \Delta} \frac{\mathrm{d} \Delta}{\mathrm{d} \lambda}=0
$$

$$
\frac{\mathrm{d} \theta_{l}}{\mathrm{~d} \lambda}=-\frac{\frac{\partial \omega}{\partial \Delta} \frac{\mathrm{d} \Delta}{\mathrm{d} \lambda}}{t+\frac{\partial \omega}{\partial \theta_{l}}}
$$

We already know that $\frac{\mathrm{d} \Delta}{\mathrm{d} \lambda}<0$. To get the signs of $\frac{\partial \omega}{\partial \Delta}$ and $\frac{\partial \omega}{\partial \theta_{l}}$, let us go back to Equation (3.6), and expand it into real and imaginary part, namely

$$
\begin{aligned}
& \rho \cos \omega=\left(1+\Delta^{2}\right) \cos \theta-2 \Delta, \\
& \rho \sin \omega=\left(1-\Delta^{2}\right) \sin \theta .
\end{aligned}
$$

Note that by Equation (3.8), $\sin \omega>0$, and partially differentiating Equation (3.7) with respect to $\Delta$ and $\theta$, we have

$$
\begin{gathered}
\frac{\partial \omega}{\partial \Delta}=-\frac{2(\Delta \cos \theta-1)}{\rho \sin \omega}>0, \\
\frac{\partial \omega}{\partial \theta}=\frac{\left(1+\Delta^{2}\right) \sin \theta}{\rho \sin \omega}>0 .
\end{gathered}
$$

Hence $\theta_{l}^{\prime}(\lambda)>0$. The claim (3.2) is thus established and the proof is complete.

3.3. Uniform trees with trunk length $m=0$. As shown by the numerical results in Table 2, this case is quite different of the previous two situations.

TheOREM 3.3. Consider a uniform tree with trunk length $m=0$. We have

$$
n_{4^{+}}\left(H_{0, k}\right) \leqslant\left\lfloor\frac{n_{\mathrm{T}}\left(H_{0, k}\right)+1}{2}\right\rfloor, \quad \forall k \geqslant 1,
$$

where $[x]$ is the largest integer no greater than $x$. Moreover, the equality is achieved when $k$ is large enough, i.e., branchs are long enough.

Proof. We proceed in two steps.

- Step 1: prove the upper bound by induction on $n_{\mathrm{T}}$.

$\diamond$ Base Case with $n_{\mathrm{T}}=2$

When $n_{\mathrm{T}}=2$, let $\widetilde{H}=(V, E \backslash\{e\})$, where $e$ connects the only two T-junctions of $H_{0}$. Then $\widetilde{H}$ consists of two paths, say $\widetilde{P}_{1}$ and $\widetilde{P}_{2}$, respectively (see Figure $5($ a)). By Equation (2.3), $\max \left\{\lambda_{1}\left(\widetilde{P}_{1}\right), \lambda_{1}\left(\widetilde{P}_{2}\right)\right\}<4$. By Lemma $2.3, \lambda_{2}\left(H_{0}\right) \leqslant \lambda_{1}(\widetilde{H})=\max \left\{\lambda_{1}\left(\widetilde{P}_{1}\right), \lambda_{1}\left(\widetilde{P}_{2}\right)\right\}<4$. Since one can cut either of the branches in $H_{0}$ to produce a starlike tree, by Lemma 2.3 this means $\lambda_{1}\left(H_{0}\right) \geqslant 4$. Hence we have $n_{4^{+}}=1 \leqslant\left\lfloor\left(n_{\mathrm{T}}+1\right) / 2\right\rfloor$. 


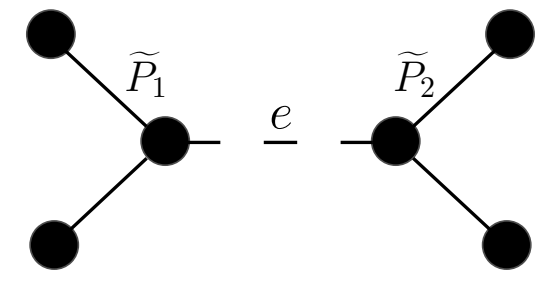

(a) Base Case 1

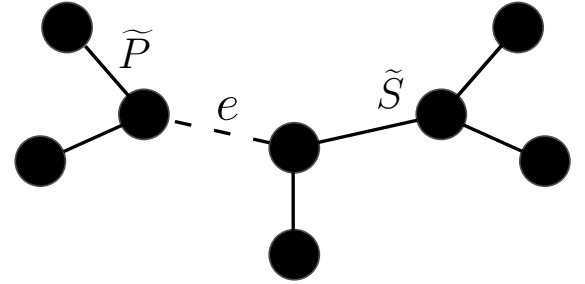

(b) Base Case 2

FIG 5. Two examples of base cases in the proof of Theorem 3.3.

$\diamond$ Base Case with $n_{\mathrm{T}}=3$

When $n_{\mathrm{T}}=3$, let $\widetilde{H}=(V, E \backslash\{e\})$, where $e$ connects either pair of the consecutive Tjunctions of $H_{0}$. Then $\widetilde{H}$ consists of one path and one starlike tree, say $\widetilde{P}$ and $\widetilde{S}$, respectively (see Figure $5(\mathrm{~b}))$. Then $\lambda_{1}(\widetilde{P})<4$ by Equation $(2.3), \lambda_{1}(\widetilde{S})>4>\lambda_{2}(\widetilde{S})$ by Lemma 2.1. Furthermore, by Lemma 2.3, $\lambda_{1}\left(H_{0}\right) \geqslant \lambda_{1}(\widetilde{H})=\max \left\{\lambda_{1}(\widetilde{P}), \lambda_{1}(\widetilde{S})\right\}=\lambda_{1}(\widetilde{S})>4$, and $\lambda_{3}\left(H_{0}\right) \leqslant \lambda_{2}(\widetilde{H})=\max \left\{\lambda_{1}(\widetilde{P}), \lambda_{2}(\widetilde{S})\right\}<4$. Hence, we have $n_{4^{+}} \leqslant 2 \leqslant\left\lfloor\left(n_{\mathrm{T}}+1\right) / 2\right\rfloor$.

$\diamond$ Induction Suppose $n_{4^{+}} \leqslant\left\lfloor\left(n_{\mathrm{T}}+1\right) / 2\right\rfloor$ holds for all $n_{\mathrm{T}} \leqslant i$, for some $i \geqslant 2$.

When $n_{\mathrm{T}}=i+1$, let $\widetilde{H}=(V, E \backslash\{e\})$, where $e$ connects either of the T-junctions at one end of $H_{0}$ and its consecutive T-junction. Then $\widetilde{H}$ consists of a path and a tree, say $\widetilde{P}$ and $\widetilde{H}_{1}$, respectively. Note that $\widetilde{H}_{1}$ has $n_{\mathrm{T}}-2$ T-junctions, and thus at most $\left\lfloor\left(n_{\mathrm{T}}-2+1\right) / 2\right\rfloor=$ $\left\lfloor\left(n_{\mathrm{T}}-1\right) / 2\right\rfloor$ eigenvalues are no smaller than 4 . Since all the eigenvalues of $P$ are less than 4 , by Lemma $2.3, n_{4^{+}} \leqslant\left\lfloor\left(n_{\mathrm{T}}-1\right) / 2\right\rfloor+1=\left\lfloor\left(n_{\mathrm{T}}+1\right) / 2\right\rfloor$.

- Step 2: prove the condition to achieve the upper bound.

We will use $t$ instead of $n_{\mathrm{T}}$ for simplicity. Following a procedure similar to the one used in the proof of Theorem 3.2 with $k \rightarrow \infty$, we get

$$
\begin{aligned}
& \lim _{k \rightarrow \infty} \operatorname{det}\left(\lambda \mathbf{I}-\mathbf{L}_{0}\right)=\operatorname{det}(\lambda \mathbf{I}-\underset{\sim}{\mathbf{B}}) \cdot \operatorname{det}\left(\begin{array}{cccccc}
\lambda-3-2 \alpha & 1 & & & & \\
1 & \lambda-3-\alpha & \ddots & & & \\
& \ddots & \ddots & \ddots & \ddots & \\
& & \ddots & \ddots & \ddots & \ddots \\
& & & \ddots & \lambda-3-\alpha & \ddots \\
& & & & 1 & \lambda-3-2 \alpha
\end{array}\right)_{t \times t} \\
& =\operatorname{det}(\lambda \mathbf{I}-\underset{\sim}{\mathbf{B}}) \cdot \prod_{l=1}^{t} \mu_{0, l}(\lambda),
\end{aligned}
$$

where $\mathbf{L}_{0}$ is a short for $\mathbf{L}\left(H_{0}\right)$, $\underset{\sim}{\mathbf{B}}$ is just the one defined previously in the proof of Theorem 3.2, and

$$
\mu_{0, l}(\lambda):=(\lambda-3-\alpha)+2 \cos \theta_{0, l}
$$


with also the same $\alpha$ defined previously, and $\theta_{0, l} \equiv \theta_{0, l}(\lambda) \in \mathbb{R}$ is given by

where

$$
\begin{cases}\mathrm{g}_{0}\left(\lambda, \theta_{0, l}\right)=0, & \text { if } \sin \theta_{0, l} \neq 0 \text { and } \lambda>4 \\ \cos \theta_{0, l}=-1, & \text { if } \sin \theta_{0, l}=0 \text { and } \lambda=4,\end{cases}
$$

$$
\mathrm{g}_{0}(\lambda, \theta):=\sin (t+1) \theta+2 \alpha \sin t \theta+\alpha^{2} \sin (t-1) \theta .
$$

Similarly restricting $\theta_{0, l} \in(0, \pi)$ as before. Differentiating $\mu_{0, l}(\lambda)$ with respect to $\lambda$ gives

$$
\frac{\mathrm{d} \mu_{0, l}}{\mathrm{~d} \lambda}=\frac{\lambda-2}{2 \sqrt{\lambda(\lambda-4)}}+\frac{1}{2}-2 \sin \theta_{0, l} \frac{\mathrm{d} \theta_{0, l}}{\mathrm{~d} \lambda} .
$$

In contrast to the proof of Theorem 3.2, this time we expect $\theta_{0, l}^{\prime}(\lambda)<0$. To prove this, let

$$
\rho_{0} \mathrm{e}^{\mathrm{i} \omega_{0}} \equiv\left(\mathrm{e}^{\mathrm{i} \theta}+2 \alpha+\alpha^{2} \mathrm{e}^{-\mathrm{i} \theta}\right)
$$

from which one can similarly derive

$$
\begin{aligned}
& \frac{\partial \omega_{0}}{\partial \alpha}=-\frac{2(\alpha \cos \theta+1)}{\rho_{0} \sin \omega_{0}}<0, \\
& \frac{\partial \omega_{0}}{\partial \theta}=\frac{\left(1+\alpha^{2}\right) \sin \theta}{\rho_{0} \sin \omega_{0}}>0,
\end{aligned}
$$

and $\mathrm{g}_{0}\left(\lambda, \theta_{0, l}\right)=0$ implies

$$
t \theta_{0, l}+\omega_{0}=\mathbb{Z} \pi
$$

Differentiating the above equation with respect to $\lambda$ eventually leads to

$$
\frac{\mathrm{d} \theta_{0, l}}{\mathrm{~d} \lambda}=-\frac{\frac{\partial \omega_{0}}{\partial \alpha} \frac{\mathrm{d} \alpha}{\mathrm{d} \lambda}}{t+\frac{\partial \omega_{0}}{\partial \theta_{0, l}}}<0 .
$$

However, similar to the proof of Theorem 3.2, we here have

$$
\mu_{0, l}\left(\frac{16}{3}\right)=2 \cos \theta_{0, l}\left(\frac{16}{3}\right)+2>0
$$

but

$$
\mu_{0, l}(4)=2 \cos \theta_{0, l}(4)=2 \cos \left(\frac{l \pi}{t}\right)
$$

So $\mu_{0, l}(4)<0$ only if $t / 2<l<t$, and it is trivial to see that there are exactly $\lfloor(t+1) / 2\rfloor$ number of such l's, which completes our proof. 
TABLE 3

Summary statistics for the two dendrite graphs. Upper block: number of eigenvalues $\geqslant 4$ as well as vertices with degree $=3$ and $\geqslant 4$. Middle block: distribution of trunk lengths $m$. Lower block: statistics of branch lengths $k$.

\begin{tabular}{crr} 
& RGC \#100 & RGC \#60 \\
\hline$n_{4^{+}}$ & 12 & 138 \\
$n_{\mathrm{T}}$ & 12 & 166 \\
$\#\{\operatorname{deg}(v) \geqslant 4\}$ & 0 & 7 \\
\hline
\end{tabular}

\begin{tabular}{ccc}
\hline$\%$ of trunks with different lengths & \\
$m=0$ & $0 \%$ & $27.3 \%$ \\
$m=1$ & $0 \%$ & $8.7 \%$ \\
$m \geqslant 2$ & $100 \%$ & $64.0 \%$ \\
\hline branch length statistics & \\
min & 15 & \\
max & 132 & 125 \\
mean & 64.8 & 20.7 \\
median & 64 & 13 \\
\hline
\end{tabular}

4. Analysis of dendrites of RGCs. In the previous sections, we have analyzed in detail how characteristic numbers such as T-junction numbers, branch and trunk lengths impact on the number of spike eigenvalues of the graph Laplacian of the class of uniform trees. The purpose of this section is to show how these theoretical results on uniform trees can help us understand the phase transition observed in dendrite graphs as shown in Figure 1.

First Table 3 gives a few summary statistics on different characteristics of the two dendrite graphs shown in Figure 1(a)-(b).

We proceed as follows.

1. Each dendrite graph is assimilated as a mixture of uniform trees $H_{m, k}$ with different trunk and branch lengths $(m, k)$;

2. Using the theoretical results established previously on the number of spike eigenvalues for uniform trees, we propose an estimate, say $\widehat{n_{4^{+}}}$, for the observed number of spike eigenvalues of the dendrite graph, say $n_{4^{+}}$;

3. By confronting these two numbers, the meaningness of uniform trees as a model for dendrite graphs can be assessed.

To start with, it is important to note the following features in a dendrite graph which do not exist in a uniform tree:

(d1) Branch lengths may vary a lot in a real dendrite graph;

(d2) Trunks that incident to the same T-junction may have different lengths;

(d3) Not all T-junctions lie along a line, i.e., there may be T-junctions whose neighboring vertices are all T-junctions;

(d4) Not all junctions are T-junctions, i.e., there may be junctions of degree larger than 3. 

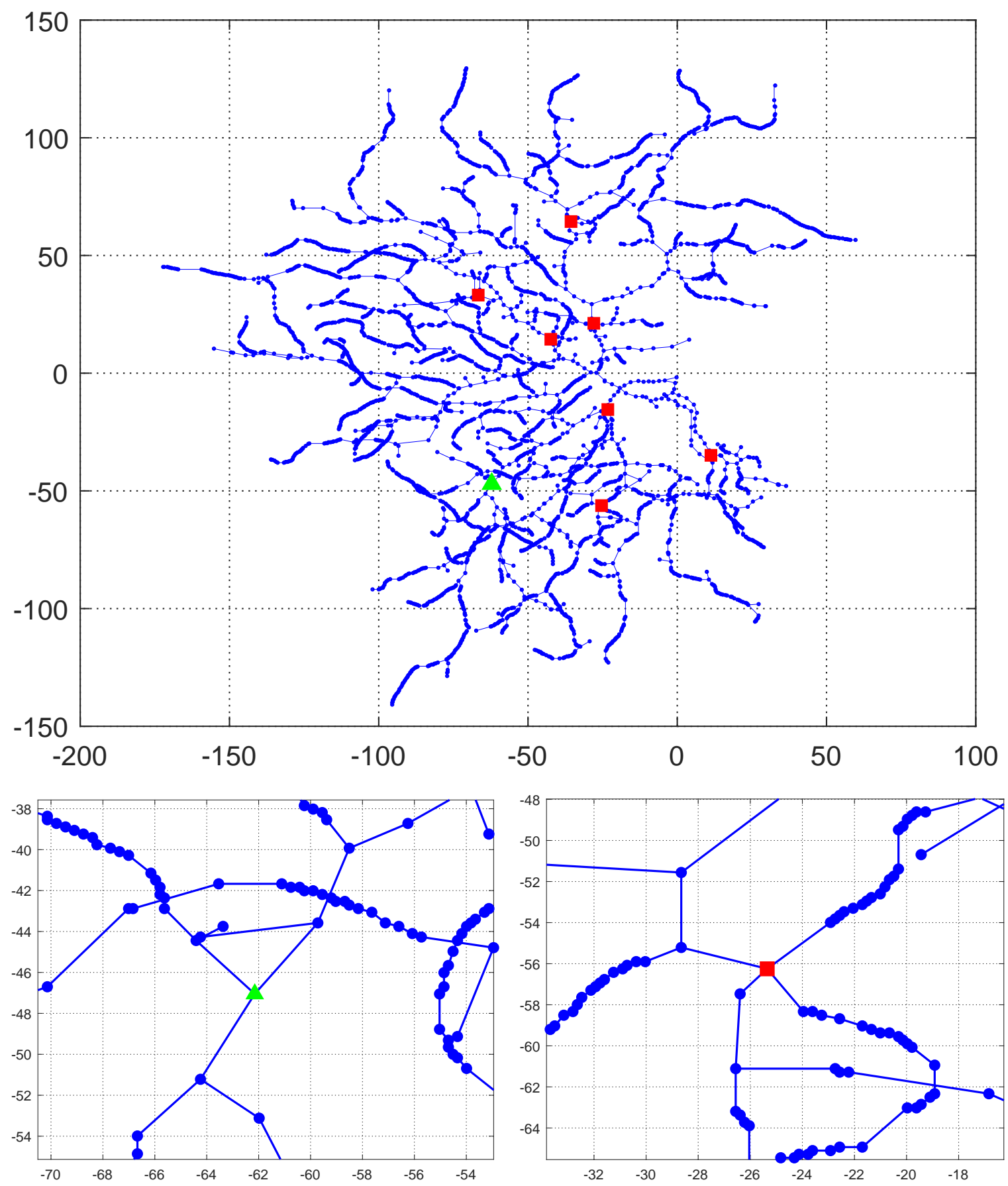

FIG 6. Upper: dendrite graph of $R G C$ \#60 with marked vertices corresponding to features (d3)(green triangle) and $(\mathrm{d} 4)$ (red square). Lower left: zoom up region around feature (d3). Lower right: zoom up region around the bottom feature (d4). 
Example of features (d3) and (d4) are given in Figure 6.

Note that from our previous discussion, $n_{4^{+}}(G)$ is non-decreasing for any dendrite graph $G$ if any of its branch is extended by one unit. Therefore, as long as branches are long enough, the limiting results derived for uniform trees still hold even though branch lengths are not constant anymore (feature (d1)). Note also from Table 3, very few vertices in those dendrite graphs have degree greater than 3 (feature $(\mathrm{d} 4)$ ). Finally, to deal with the remaining features (d2)-(d3), we propose the following method of estimation for the number of spike eigenvalues $n_{4^{+}}$.

Estimating the number of spike eigenvalues $\mathbf{n}_{\mathbf{4}^{+}}$. Let $G=(V, E)$ be a dendrite graph and consider the set of junction vertices

$$
J:=\{v \in V \mid \operatorname{deg}(v) \geqslant 3\}
$$

(i) For each junction vertex $v \in J$, find all trunks incident at $v$ (recall that a trunk is a path connecting two junction vertices). Let $m(v)$ be the minimum of the lengths of these incident trunks. Next classify $v$ into one of the three groups $\left\{J_{0}, J_{1}, J_{2^{+}}\right\}$according to the conditions $m(v)=0, m(v)=1$ and $m(v) \geqslant 2$, respectively.

(ii) The estimate for the number of spike eigenvalues $n_{4^{+}}$is

$$
\widehat{n_{4^{+}}}=\left\lfloor\frac{n_{0}+1}{2}\right\rfloor+n_{1}+n_{2^{+}}
$$

where $\left(n_{0}, n_{1}, n_{2^{+}}\right):=\left(\# J_{0}, \# J_{1}, \# J_{2^{+}}\right)$.

Note that the formula in (4.1) is directly inspired by the theoretical results for uniform trees presented in Theorems 3.1, 3.2 and 3.3. Applying this estimator to the two dendrite graphs shown on Figure 1, we obtain $\widehat{n_{4^{+}}}=12$ and 134 for RGC \#100 and RGC \#60, respectively. These predicted values are very close to their true value $n_{4^{+}}$in Table 3 , namely $n_{4^{+}}=12$ and 138 for RGC \#100 and RGC \#60, respectively. In conclusion, viewing a dendrite graph as a mixture of uniform trees of different types can help one predict the number of spike eigenvalues of the graph Laplacian.

Acknowledgments. We sincerely thank Professor N. Saito for providing us with data of retinal ganglion cells \#60 and \#100 used in this paper and the Matlab code for drawing Figure 1. We are also grateful to Ms. Yihui Ma who contributed to a preliminary draft of the paper.

\section{REFERENCES}

[1] J. Coombs, D. van der List, G.-Y. Wang and L. M. Chalupa (2006) Morphological properties of mouse retinal ganglion cells. Neuroscience, 140 : 123-136. 
[2] Y. Nakatsukasa, N. Saito, E. Woei (2013). Mysteries around the graph Laplacian eigenvalue 4. Linear Algebra and Its Applications, 438 (8):3231-3246.

[3] N. Saito and E. Woei (2009). Analysis of neuronal dendrite patterns using eigenvalues of graph Laplacians. Japan Society for Industrial and Applied Mathematics Letters, $1: 12-16$.

[4] R. Grone, R. Merris, V.S. Sunder (1990). The Laplacian spectrum of a graph. SIAM Journal on Matrix Analysis and Applications, $11: 218-238$.

[5] W. C. Yueh and S. S. Cheng (2008). Explicit eigenvalues and inverses of tridiagonal Toeplitz matrices with four perturbed corners. The ANZIAM Journal, 49(3):361-387.

Department of Statistics and Actuarial Science The University of Hong Kong

E-MAIL: xuyy@connect.hku.hk; jeffyao@hku.hk 